\title{
Temporary cardiac pacing via the oesophagus
}

\author{
DAVID N. COOPER \\ M.B., Ch.B., M.R.C.P. (U.K.) \\ Department of Medicine, Hope Hospital, Salford
}

\begin{abstract}
Summary
Two cases are reported in which temporary cardiac pacing was performed using an oesophageal electrode. The benefits and disadvantages of this technique are discussed.
\end{abstract}

\section{Introduction}

Temporary transvenous pacing is routinely used for patients with drug-resistant symptomatic bradycardia complicating acute myocardial infarction. The technique requires skill and facilities which are not always immediately available in district general hospitals. Temporary pacing using an oesophageal electrode is a simple and effective alternative as illustrated by the following cases.

\section{Case 1}

A 64-year-old man was admitted to the Coronary Care Unit with a 2-hr history of severe retrosternal chest pain and electrocardiographic changes of a recent inferior myocardial infarction. Eight hours after admission he went into ventricular fibrillation which was successfully treated with a single DC shock. Sinus rhythm was restored but he had numerous ventricular ectopics which were suppressed by i.v. disopyramide. Twenty-four hours after admission he complained of severe chest pain and became shocked. His electrocardiogram showed a 'wandering atrial pacemaker' and a ventricular rate of $30 / \mathrm{min}$. The disopyramide was stopped and atropine sulphate $1.8 \mathrm{mg}$ was given i.v. without effect. Pending transfer to the screening room for transvenous pacing his respiration ceased and he was intubated and ventilated. He was not thought fit to be moved from the Coronary Care Unit and therefore a bipolar oesophageal pacing electrode (Vygon) was passed to about $35 \mathrm{~cm}$ via the nose. Effective pacing was established with a voltage of 25 at a fixed rate of $70 / \mathrm{min}$. This was followed by rapid improvement in his condition with a rise in his blood pressure to $100 / 60 \mathrm{mmHg}$. He made a satisfactory recovery but required pacing for $72 \mathrm{hr}$ before reverting to sinus rhythm. During this period he had no discomfort or dysphagia. Ninety-six hours after insertion, the electrode was removed. He was discharged 13 days after admission.

\section{Case 2}

A 75-year-old woman was admitted to hospital after a 4-hr history of chest pain and electrocardiogram changes of a recent inferior infarction. She had an unstable rhythm with atrial fibrillation, nodal rhythm and atrio-ventricular block with a bradycardia of $40-50 / \mathrm{min}$. Her general condition was poor with an unrecordable blood pressure, Cheyne-Stokes respiration and confusion. A bipolar electrode was introduced into the oesophagus through the nose and fixed rate trans-oesophageal pacing using $25 \mathrm{~V}$ was successfully established. This resulted in a rapid improvement in her blood pressure, respiration and mental state. She was paced for $90 \mathrm{~min}$ and then a transvenous pacemaker was inserted and the oesophageal electrode removed. She required pacing for a further 5 days.

\section{Discussion}

Trans-oesophageal cardiac pacing was originally studied by Shafiroff and Linder (1957) and has subsequently been shown not to cause damage to the oesophageal mucosa (Burack and Furman, 1969; Lubell, 1971). The first clinical application was by Burack and Furman but since then there have been only 2 reports in the literature regarding its successful use in the management of dangerous bradyarrhythmias. The technique of trans-oesophageal cardiac pacing has been described elsewhere (Hale et al., 1980). The Vygon system used in these cases comprises a bipolar oesophageal electrode in conjunction with an amplifier and standard pacing box. The procedure is safe, simple and short, requiring neither special skills, aseptic technique nor $X$-ray screening. It can be valuable in the emergency management of dangerous bradyarrhythmias complicating myocardial infarction, as these cases illustrate, but owing to limited experience, its reliability for prolonged pacing has not yet been established. Its potential disadvantages include failure of capture, oesophageal discomfort and 
dysphagia, and skeletal muscle stimulation. On the basis of published reports and the present authors' experience, the latter 2 disadvantages have not been a problem provided a voltage of less than 40 is used.

\section{Acknowledgments}

I thank Dr D. Gaon and Dr H. Cohen for allowing me to report the cases and Dr Gaon for his help in preparing the paper. I also thank Kim Lindley for her secretarial assistance.

\section{References}

Burack, B. \& Furman, S. (1969) Transesophageal cardia® pacing. American Journal of Cardiology, 23, 469.

Hale, J., Kelly, A.J., Bowes, R.J., Ilchyshyn, A. Ellenbosen, E.K. (1980) Emergency transoesophageaEs cardiac pacing. Cardiovascular Technology, 22, 38.

LUBELL, D.L. (1971) Cardiac pacing from the esophagus? American Journal of Cardiology, 27, 641.

Shafiroff, G.B.P. \& Linder, J. (1957) Effects of externa $\overline{\bar{h}}$ electrical stimuli on the human heart. Journal of Thoraci $\mathbb{C}$ Surgerv, 33, 544. 